Didaktik der Informatik 
Sigrid Schubert Andreas Schwill

\title{
Didaktik der Informatik
}

\author{
2. Auflage
}




\author{
Autoren \\ Prof. Dr. Sigrid Schubert, Universität Siegen \\ E-Mail: sigrid.schubert@uni-siegen.de \\ Prof. Dr. Andreas Schwill, Universität Potsdam \\ E-Mail: schwill@cs.uni-potsdam.de
}

\begin{abstract}
Wichtiger Hinweis für den Benutzer
Der Verlag und die Autoren haben alle Sorgfalt walten lassen, um vollständige und akkurate Informationen in diesem Buch zu publizieren. Der Verlag übernimmt weder Garantie noch die juristische Verantwortung oder irgendeine Haftung für die Nutzung dieser Informationen, für deren Wirtschaftlichkeit oder fehlerfreie Funktion für einen bestimmten Zweck. Ferner kann der Verlag für Schäden, die auf einer Fehlfunktion von Programmen oder ähnliches zurückzuführen sind, nicht haftbar gemacht werden. Auch nicht für die Verletzung von Patent- und anderen Rechten Dritter, die daraus resultieren. Eine telefonische oder schriftliche Beratung durch den Verlag über den Einsatz der Programme ist nicht möglich. Der Verlag übernimmt keine Gewähr dafür, dass die beschriebenen Verfahren, Programme usw. frei von Schutzrechten Dritter sind. Die Wiedergabe von Gebrauchsnamen, Handelsnamen, Warenbezeichnungen usw. in diesem Buch berechtigt auch ohne besondere Kennzeichnung nicht zu der Annahme, dass solche Namen im Sinne der Warenzeichen- und Markenschutz-Gesetzgebung als frei zu betrachten wären und daher von jedermann benutzt werden dürften. Der Verlag hat sich bemüht, sämtliche Rechteinhaber von Abbildungen zu ermitteln. Sollte dem Verlag gegenüber dennoch der Nachweis der Rechtsinhaberschaft geführt werden, wird das branchenübliche Honorar gezahlt.
\end{abstract}

\title{
Bibliografische Information der Deutschen Nationalbibliothek
}

Die Deutsche Nationalbibliothek verzeichnet diese Publikation in der Deutschen Nationalbibliografie; detaillierte bibliografische Daten sind im Internet über http://dnb.d-nb.de abrufbar.

Springer ist ein Unternehmen von Springer Science+Business Media springer.de

2. Auflage 2011

(C) Spektrum Akademischer Verlag Heidelberg 2011

Spektrum Akademischer Verlag ist ein Imprint von Springer
$\begin{array}{lllll}11 & 12 & 13 & 14 & 15\end{array}$
$\begin{array}{lllll}5 & 4 & 3 & 2 & 1\end{array}$

Das Werk einschließlich aller seiner Teile ist urheberrechtlich geschützt. Jede Verwertung außerhalb der engen Grenzen des Urheberrechtsgesetzes ist ohne Zustimmung des Verlages unzulässig und strafbar. Das gilt insbesondere für Vervielfältigungen, Übersetzungen, Mikroverfilmungen und die Einspeicherung und Verarbeitung in elektronischen Systemen.

Planung und Lektorat: Dr. Andreas Rüdinger, Barbara Lühker

Herstellung: Crest Premedia Solutions (P) Ltd, Pune, Maharashtra, India

Umschlaggestaltung: SpieszDesign, Neu-Ulm

Titelbild: C SpieszDesign

Satz: Autorensatz

ISBN 978-3-8274-2652-9 


\section{Vorwort}

Aus dem Vorwort zur 1. Auflage 2004: „Informatik ist bereits seit mehreren Jahrzehnten mehr oder weniger weit in die Schulen vorgedrungen. Dennoch besteht immer noch ein sehr großer Bildungsbedarf auf dem Gebiet „Didaktik der Informatik“, der zum einen historisch entstanden ist aus dem fortwährenden Mangel an grundständig ausgebildeten Informatiklehrkräften und dem positiven Phänomen der Enthusiasten, die sich autodidaktisch in die Informatik einarbeiteten und so den Fortbestand einer attraktiven, wenn auch sehr subjektiv geprägten informatischen Bildung sicherten. Zum anderen ist es die Informatik selbst, die mit ihrer dynamischen Entwicklung ständig eine Beobachtung, Bewertung und Neukonzeption unterrichtlicher Zugänge erforderlich macht. Während aber vor wenigen Jahren der Bereich „Didaktik der Informatik“ noch durch eine Ansammlung von exemplarischen Einzellösungen und Unterrichtsbeispielen geprägt war, für die dann kein systematischer Überbau vorlag, ist in der Zwischenzeit eine Forschungs-Community entstanden, die zentrale Fragestellungen der Informatikdidaktik bearbeitet und auf deren Ergebnisse verwiesen werden kann. Mit diesem Buch wird daher auch ein Beitrag zur Orientierung des wissenschaftlichen Nachwuchses geleistet."

Während die im letzten Jahrhundert entstandenen wissenschaftlichen Arbeiten vor allem konzeptionell geprägt und auf eine theoretische Fundierung der Schulinformatik ausgerichtet waren, z.B. das Konzept der fundamentalen Ideen, das Konzept des informationszentrierten Zugangs, das Konzept der didaktischen Systeme, das Konzept der Dekonstruktion, ist in den letzten Jahren eine Reihe von wissenschaftlichen Arbeiten entstanden, die empirische Absicherungen von didaktischen Ansätzen und Sichtweisen beigetragen haben. Nun können informatikdidaktische Empfehlungen für die Grundbildung und Vertiefung im Unterrichtsfach Informatik auch begründet werden. Im vorliegenden Buch nimmt diese Diskussion breiten Raum ein und schließt auch kritische Sichtweisen zu den Vor- und Nachteilen unterrichtlichen Handelns ein. Dabei ist es ein wesentliches Anliegen des Buches, über die Vielfalt und den geistigen Reichtum der Informatik aufzuklären und beispielhaft zu zeigen, wie die Transformation in den Lernprozess der Schülerinnen und Schüler erfolgen kann, anstatt genau einen Weg zur Vermittlung von Informatik vorzuschlagen.

Dieses Buch wendet sich an alle Studierenden des Lehramts Informatik, an alle Referendarinnen und Referendare, die das Schulfach Informatik unterrichten, und an alle Informatiklehrerinnen und -lehrer, die an Unterrichtskonzepten für 


\section{vi | Vorwort}

die informatische Bildung und an unterrichtspraktischen Auswirkungen neuer Forschungsergebnisse interessiert sind.

Gegenüber der 1. Auflage sind fünf neue Kapitel hinzugekommen; das Buch gliedert sich damit in 16 Kapitel, von denen die ersten drei das junge Fachgebiet „Didaktik der Informatik“" theoretisch untersetzen.

Das Kapitel „Problemlösen“ zeigt die ausgeprägten Problemlöse-Strategien und liefert Beispiele über den Zugang der logischen Programmierung. Dem neueren Ansatz, Unterricht nicht mehr lernzielorientiert zu planen, sondern an Kompetenzen auszurichten, wird im Kapitel „Kompetenzentwicklung“ breiter Raum gewidmet. Die Kapitel „Informatisches Modellieren und Konstruieren“, „Objektorientierte Denkweisen“, „Strukturen untersuchen und Strukturieren“ und „Sprachen, Automaten und Netze“ vertiefen die empfohlenen Bildungsschwerpunkte und untersetzen diese mit Lernaufgaben. Die Kapitel „Anfangsunterricht“ und „Projektunterricht" geben Anregungen für zwei besonders typische, aber auch besonders schwierige Phasen informatischer Bildung. Das Kapitel „Unterrichtshilfen für den Informatikunterricht" ist der Nutzung aller im Informatikunterricht bekannten Hilfsmittel gewidmet; dazu zählt in erster Linie der Rechner selbst, mehr und mehr finden aber auch Modelle große Aufmerksamkeit, die mechanisch oder unter Einbindung des Lernenden selbst informatische Sachverhalte zu veranschaulichen suchen. Oft erfolgt der Zugang Jugendlicher zu Informatiksystemen über das Internet; damit nicht nur Bedienungsfertigkeit, sondern auch Verständnis der komplexen Zusammenhänge erworben wird, sind besondere Zugänge nötig, die in einem Kapitel „Internetworking" behandelt werden.

Die beiden letzten Kapitel von den Gastautoren Peer Stechert und Ralf Romeike vertiefen zwei Aspekte informatischer Ausbildung, in denen die beiden besondere Expertise erworben haben. Peer Stechert berichtet von einem Unterrichtsprojekt zur Förderung der Kompetenzentwicklung mit Informatiksystemen in der Schulpraxis, Ralf Romeike zeigt, wie im Unterrichtsfach Informatik kreative Prozesse, z.B. durch Modellierungstätigkeiten, gefördert werden können.

Wir danken den beiden Gastautoren Ralf Romeike und Peer Stechert ganz herzlich für ihre spontane Zusage; ein besonderer Dank auch an Christian Eibl, der mit großem technischen Geschick das Gesamtlayout des Buches erstellt hat.

Viel Spaß beim Lesen!

Siegen und Potsdam, im Frühjahr 2011

Sigrid Schubert und Andreas Schwill 


\section{Inhaltsverzeichnis}

1 Didaktik der Informatik ....................................................... 1

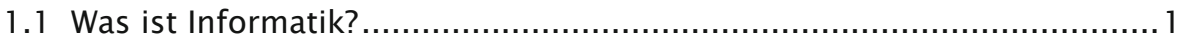

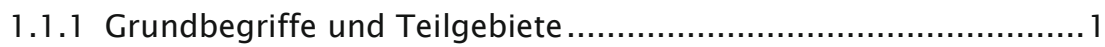

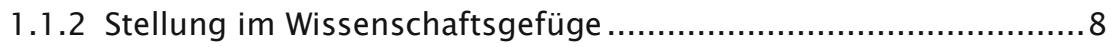

1.2 Didaktik der Informatik und ihre Geschichte .................................... 12

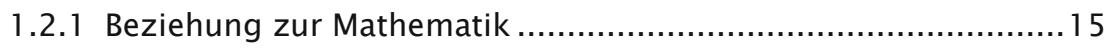

1.2.2 Unterrichtsziele und historische didaktische Ansätze ................ 17

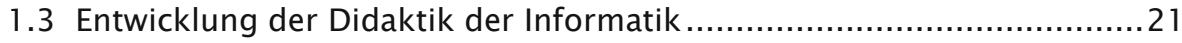

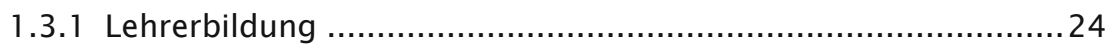

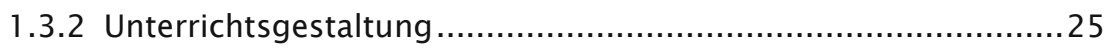

2 Grundmodell für Ziele, Inhalte und Lehrmethoden..................... 31

2.1 Informatikdidaktische Orientierung für Lehrer und Schüler ...................31

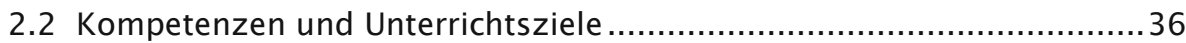

2.3 Auswahl und Klassifikation der Unterrichtsinhalte..............................40

2.4 Gestaltung und Bewertung typischer Unterrichtssituationen .................44

2.5 Fachübergreifendes und fächerverbindendes Lernen ..........................50

3 Theoretische Fundierung der Schulinformatik .......................... 53

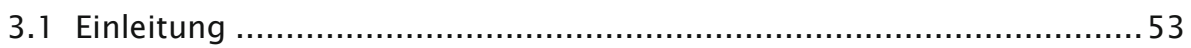

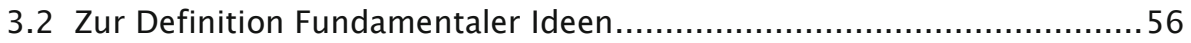

3.2.1 Der Ideenbegriff in der Philosophie..........................................56

3.2.2 Der Begriff der Fundamentalen Ideen in der Pädagogik .............59

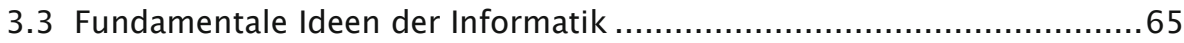

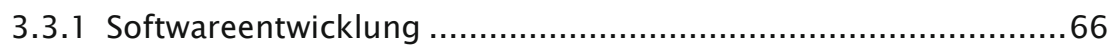

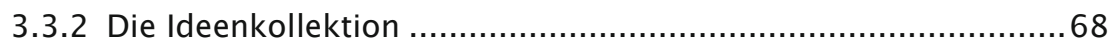

3.3.3 Modellbildung und Masterideen............................................. 75

3.3.4 Fundamentale Ideen als Konzept- und Relevanzfilter .................76

3.4 Fundamentale Ideen - Bestandsaufnahme und Weiterentwicklungen ....77

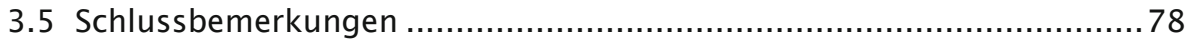

4 Problemlösen im Informatikunterricht ................................... 81

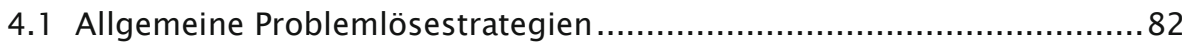




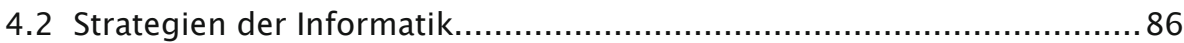

4.3 Unterrichtsbeispiele ..................................................... 91

5 Kompetenzentwicklung ................................................ 111

5.1 Entwicklung von Basiskompetenzen ...................................... 111

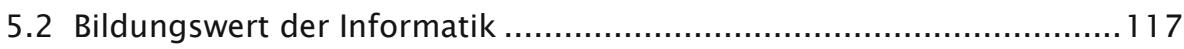

5.3 Bildungsstandards und Kompetenzmodelle .............................. 118

5.4 Wissen strukturieren ....................................................... 123

5.5 Von Aufgaben zu Aufgabenklassen ...................................... 125

5.6 Lernen durch Exploration ................................................... 129

5.7 Handlungsmuster.......................................................... 131

5.8 Evaluation des Informatikunterrichts .................................... 132

6 Informatisches Modellieren und Konstruieren......................... 135

6.1 Der Modellbegriff in der Informatik .................................... 135

6.2 Spezifikation ................................................................. 141

6.3 Daten- und Ablaufmodellierung ........................................ 144

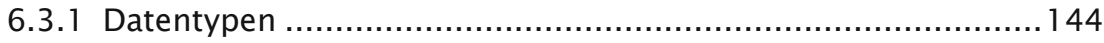

6.3 .2 Ablaufstrukturen ................................................ 148

7 Objektorientierte Denkweisen............................................. 157

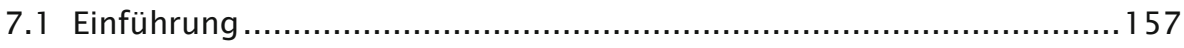

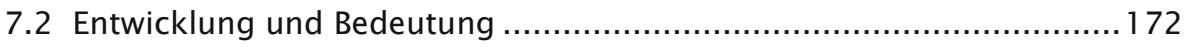

7.3 Vertiefung in Informatik mit OOM...................................... 176

8 Unterrichtshilfen für den Informatikunterricht..................... 187

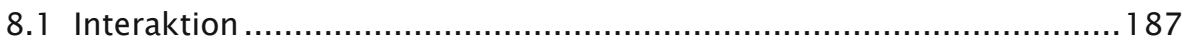

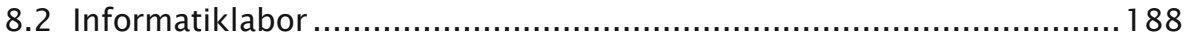

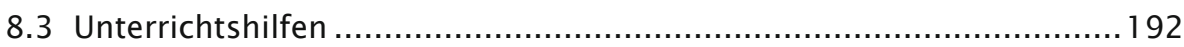

8.3.1 Historische und kognitionspsychologische Notizen zur Anschauung ......................................................... 193

8.3.2 Theorie und Klassifikation von Unterrichtshilfen ................... 196

8.3.3 Beispiele für Unterrichtshilfen .................................... 196

8.4 Experimente im Informatikunterricht ........................................ 200

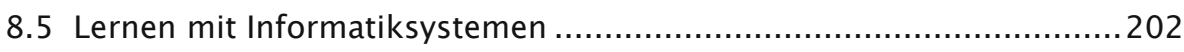

9 Informatiksysteme ............................................................. 213

9.1 Wirkprinzipien von Informatiksystemen ...................................... 213

9.2 Reale, abstrakte und virtuelle Maschine ................................... 214

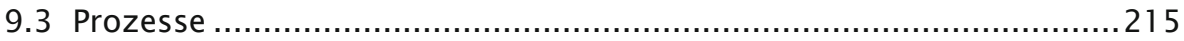

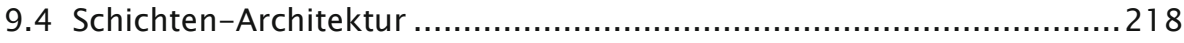


10 Strukturen untersuchen und Strukturieren ............................ 235

$10.1 \mathrm{Gl}$-Empfehlungen........................................................... 235

10.1.1 Gesamtkonzept zur informatischen Bildung .....................235

10.1.2 Bildungsstandards Informatik ...................................236

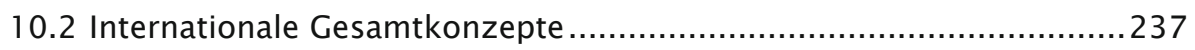

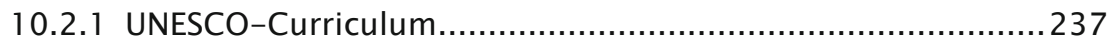

10.2.2 A Model Curriculum for K-12 Computer Science ..................238

10.3 Strukturieren in einem Gesamtkonzept...................................240

10.3.1 Informatische Bildung............................................. 240

10.3.2 Daten und Modelle .................................................. 242

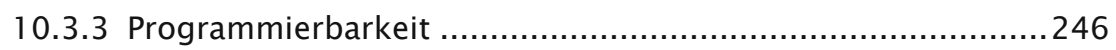

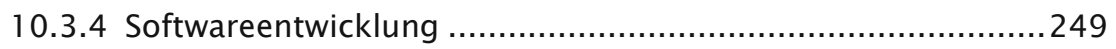

11 Sprachen, Automaten und Netze ......................................... 253

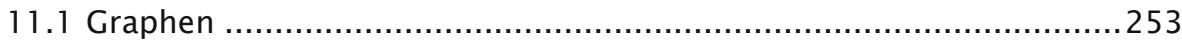

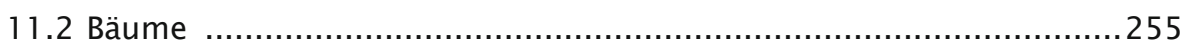

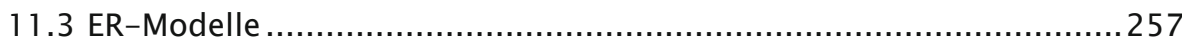

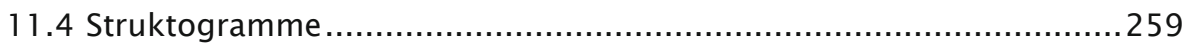

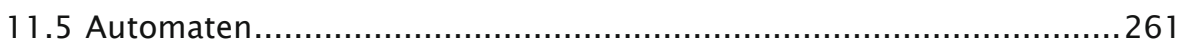

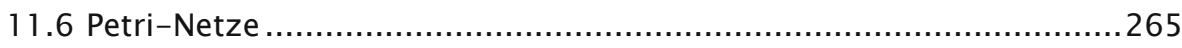

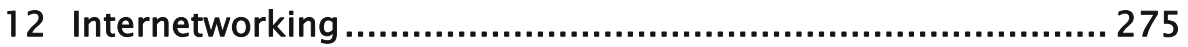

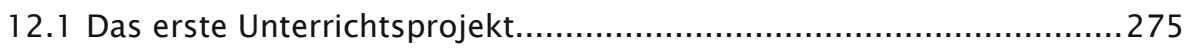

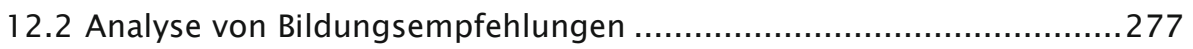

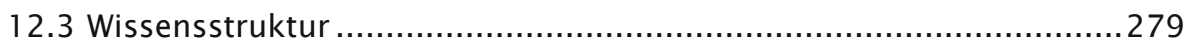

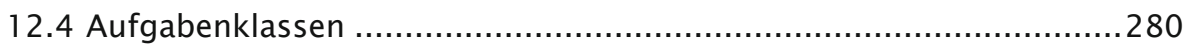

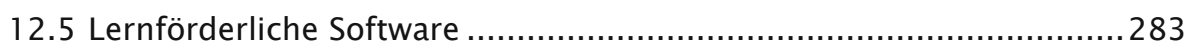

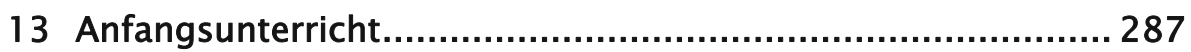

13.1 Der programmiersprachliche Zugang..................................... 289

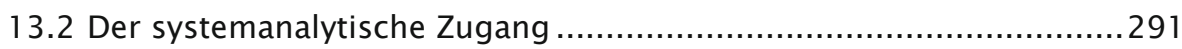

13.3 Der Zugang über Lernumgebungen .....................................293

13.4 Kognitive Aspekte objektorientierter Programmierung .....................295

13.5 Der projektorientierte fächerübergreifende Zugang .....................297

13.6 Mädchen und Jungen im Fach Informatik ...............................298

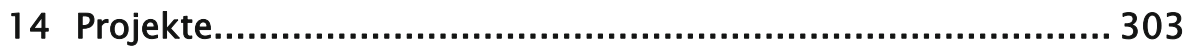

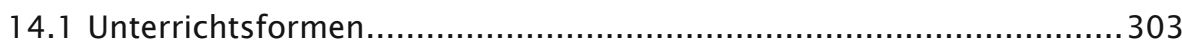

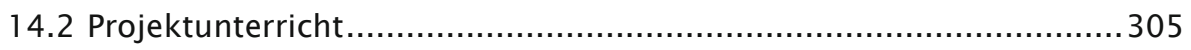


14.2.1 Pädagogische Aspekte des Projektunterrichts .....................305

14.2.2 Informatische Aspekte des Projektunterrichts .....................308

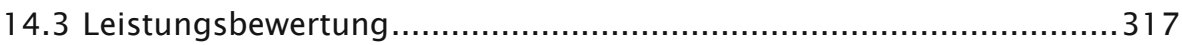

14.4 Projektbeispiel: Keywords in Context (KWIC) ............................... 319

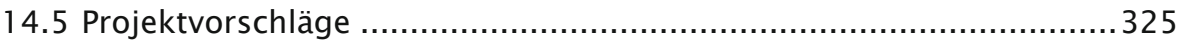

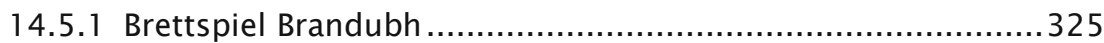

14.5.2 Game of Life im fächerübergreifenden Unterricht mit Biologie............................................................ 327

15 Kompetenzentwicklung mit Informatiksystemen ................... 333

15.1 Unterrichtsmodell KIS...................................................... 334

15.2 Rahmenbedingungen des Unterrichts ........................................ 335

15.2.1 Zielgruppenspezifische Planung .....................................335

15.2.2 Unterrichtsmethodik und technischer Rahmen ....................339

15.3 Lernphasen und Problemstellen im Unterricht.............................340

15.3.1 Zugriffskontrolle ................................................... 340

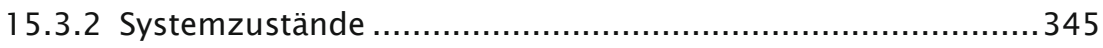

15.3.3 Kritische Betrachtung von Entwurfsmustern im Informatikunterricht der Sekundarstufe II ..............................349

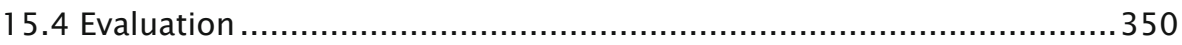

15.4.1 Auswertung der Lernerfolgskontrolle ..............................350

15.4.2 Schriftliche Akzeptanzbefragung der Schüler ......................351

15.4.3 Fazit der verantwortlichen Informatiklehrperson ..................351

15.5 Zusammenfassung und Fazit............................................. 352

16 Kreativität im Informatikunterricht ................................... 355

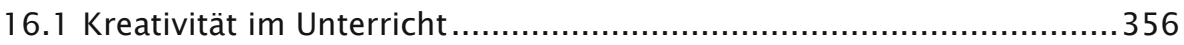

16.2 Kreatives als konstruktionistisches Lernen.................................... 356

16.3 Ansatzpunkte einer kreativen Informatik ................................ 358

16.3.1 Informatik - ein kreatives Fach ................................ 358

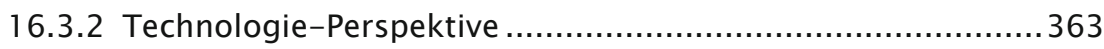

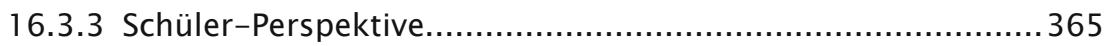

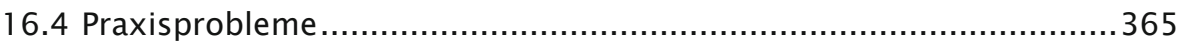

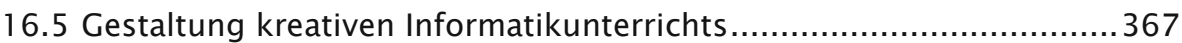

16.5.1 Der Challenge-Cycle............................................ 368

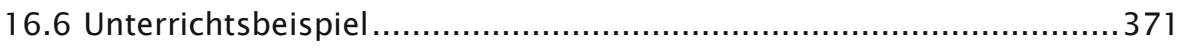

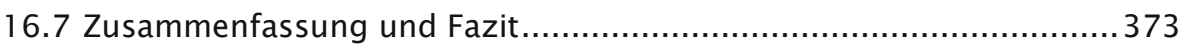


A Kompetenzbegriff der Kultusministerkonferenz 399

B Programmbeispiele 400

Ausgewählte Prolog-Beispiele 400

Ausgewählte Python-Beispiele. 403

Ausgewählte Java-Beispiele. 407 